\title{
Die Spur des Magiers Petese
}

In einer kürzlich veröffentlichten demotischen Erzählung, dem pCarlsberg 165 (mit zwei weiteren Parallelhandschriften), spielt ein Magier namens Petese, Sohn des Petatum die Hauptrolle $\left({ }^{1}\right)$. Ziel des hier vorliegenden Beitrages ist es, auf andere Belege hinzuweisen, in denen ein Magier bzw. Weiser namens Petese (oder daraus ableitbare Namensformen) auftritt. Hieran soll sich dann die Frage anschließen, inwieweit sich so etwas wie eine Tradition um eine konkrete Person fassen läßt.

Zunächst sei zur Erinnerung noch einmal kurz die Person des Petese skizziert, wie sie sich nach der demotischen Erzählung darstellt $\left({ }^{2}\right)$. Petese ist der Sohn des Petatum, des Propheten von Heliopolis. Seine Geburt scheint nicht ganz unproblematisch zu sein, jedenfalls deutet sich so etwas wie eine komplexe Ehebruchsgeschichte an, deren Details leider infolge schlechter Texterhaltung relativ dunkel bleiben. Wo er selbst handelnd in Erscheinung tritt, ist er Priester im Tempel des Re von Heliopolis. Er ist imstande, einen im Hof seines Hauses erscheinenden Geist magisch einzufangen und dazu zu zwingen, ihm die Wahrheit über geheime und verborgene Dinge zu sagen. Vor allem erfährt er dadurch, daß er nur noch 40 Tage zu leben hat. Zunächst will er für ein angemessenes Begräbnis sorgen und verlangt deshalb von den Priestern des Tempels fünfhundert Silberstücke. Als Entgelt will er geheime Schriften zugänglich machen. Die übrigen stimmen zu, der für die Finanzverwaltung zuständige Lesonispriester Hareus weigert sich jedoch.

Um ihn zu einer Meinungsänderung zu bringen, setzt Petese magische Mittel ein: Er bildet aus Wachs eine Katze und einen Falken, die magisch belebt werden. So begeben sich die Kreaturen zum Haus des Hareus, wo sie anscheinend in Kampf geraten, wobei die Katze den Falken tötet $\left(^{3}\right)$.

(1) K. Ryholt, The Story of Petese, Son of Petetum and Seventy other Good and Bad Stories, CNI Publications 23, Kopenhagen 1999.

(2) Hierbei greife ich nicht nur auf Ryholts Übersetzung zurück, sondern benutze eine in einigen Punkten verbesserte Fassung, die im Rahmen einer von Friedhelm Hoffmann und mir vorbereiteten Anthologie der demotischen Literatur erscheinen wird.

(3) Hier könnte eine symbolische Bedeutung zugrunde liegen: Petese ist Prophet des Gottes Re, der u.a. in tierischer Form als "großer Kater " verstanden wird. Hareus ist über seinen Namen dem Gott Horus zugeordnet, dessen Tier der Falke ist. So kann der Sieg der Katze über den Falken auch eine direkte Drohung beinhalten. 
Voller Schrecken über diese machtvolle Magie stimmt Hareus nicht nur dem Geldverlangen des Petese zu, sondern zahlt aus eigener Tasche zusätzlich nochmals dieselbe Summe.

Nach diesen Auseinandersetzungen kann Petese sich in Ruhe seinen weiteren Vorbereitungen widmen. Erneut wird er magisch tätig, indem er aus Wachs weitere Gestalten erschafft, die magisch belebt werden. Konkret handelt es sich, neben einigen im Papyrus nicht erhaltenen Personen um zwei Vorlesepriester, einen Schreiber des Gottesbuches, vier Türhüter sowie noch Träger der Bahre (?). Diese sind für die korrekten Rituale der Bestattung zuständig, wobei sie alles so ausführen sollen, wie es für einen Fürsten oder sogar Pharao selbst gemacht wird. Femer werden zwei Paviane ebenfalls aus Wachs geschaffen und magisch belebt. Sie werden ausgeschickt, um 35 gute und 35 böse Geschichten zu sammeln und aufzuschreiben, mit denen sich Petese in den verbleibenden Tagen seines Lebens unterhalten läßt. Ferner erhalten die magisch belebten Wesen den Auftrag, ein bestimmtes Objekt (eventuell Peteses Bücher) zu verstecken, so $\mathrm{daB}$ sie nie wieder auf Erden sichtbar werden sollten. Schließlich scheint Petese sich selbst beim Eintritt seines Todes in Mumienbinden zu wickeln. Seine Frau, die ihn vermißt und denkt, er würde nur Medikamente gegen seine Krankheit nehmen, bringt seinen Anweisungen gemäß ein Räucheropfer von Weihrauch, Myrrhe und Kyphi dar. Dann spricht sie durch die geschlossene Zimmertür mit ihm. Darauf antwortet ihr der Sonnengott Re mit der Stimme des Petese. Der Rest der Erzählung ist nicht mehr zusammenhängend verständlich, so daß unsicher bleibt, ob Petese etwa durch die Gnade des Gottes wieder auf die Erde zurückkehren kann.

Die Haupthandschrift dieser Erzählung, der pCarlsberg 165 + PSI inv. D $4+$ pCtYBR 4514, stammt etwa aus dem 2. Jhd. n. Chr., ebenso wie eine weitere, der pCarlsberg $389+$ PSI Inv. D $3\left({ }^{4}\right)$. Dagegen führt die dritte Handschrift, der pSaqqara $4\left(^{5}\right)$, bereits um einiges weiter zurück. Er dürfte etwa aus dem 4. Jhd. v. Chr. stammen $\left({ }^{6}\right)$. Noch ein Jahrhundert weiter zurück kann man durch ein indirektes Zeugnis kommen. Unter den 35 guten und bösen Geschichten, mit denen Petese sich unterhalten läßt, sind inzwischen im pCarlsberg 324 Reste identifiziert worden, die eine

(4) RyHolT, Story of Petese, S. 1-11.

(5) Ursprünglich ediert von H. Smith, J.W. TalT, Saqqara Demotic Papyri, I (P. Dem. Saq. I), TE 7, London 1983, S. 149-153, T. 11 a.

(6) SMTh/T AIT, Saqqara Demotic Papyri I, S. xf. 
demotische Version der Pheros-Legende darstellen ( $\left.{ }^{7}\right)$. Da diese bereits von Herodot (II, 111) um die Mitte des fünften Jahrhunderts v. Chr. überliefert wird $\left(^{8}\right)$, besteht zumindest die Möglichkeit, auch den Kern der Rahmenhandlung um den Magier Petese mindestens nochmals um ein Jahrhundert vor der ältesten erhaltenen Handschrift anzusetzen. Dieser Ansatz ist allerdings insofern unsicher, als die Pheros-Legende auch unabhängig von der Rahmenhandlung zirkuliert haben kann.

Festzuhalten sind als generelle Merkmale des Petese sein Name mit Filiation, seine Zuordnung zum Kult des Re von Heliopolis sowie seine beachtlichen Fähigkeiten im Bereich arkaner Kenntnisse. Diese Kriterien sind sämtlich einzeln von nur begrenztem Wert, da sie häufiger auftreten können. Gerade der Name selbst ist in Ägypten alles andere als selten. Dennoch besteht die Hoffnung, durch kombiniertes Auftreten mehrerer Indizien mögliche Zusammenhänge aufdecken zu können.

Am Anfang kann noch eine weitere ägyptische Quelle stehen, die ihrer Datierung nach sogar etwas vor der oben erwähnten demotischen Erzählung liegt. In einem abnormal-hieratischen Papyrus etwa aus dem 8.-7. Jhd. v. Chr. befindet sich ein halb literarisch, halb juristisch scheinender Text, dessen einer Protagonist ein Petese aus Heliopolis ist $\left({ }^{9}\right)$. Im erhaltenen Text übt er allerdings keine magischen Handlungen aus, sondern agiert rein als Beamter. Auffällig ist aber sein Titel, nämlich p3 hri «das Oberhaupt». Diese Bezeichnung ist kein regulärer Rang- oder Funktionstitel der ägyptischen Hierarchie. Vielmehr findet sie sich zum einen als Bezeichnung eines Vorgesetzten - gleich welcher Art - besonders in ägyptischen Lehrtexten, die korrektes Verhalten gegenüber dem Vorgesetzten unabhängig von der spezifischen Situation lehren. Zum anderen ist

(7) Information von Kim Ryholt. Der pCarlsberg 324 ist nach seinen Erkenntnissen tatsächlich die Fortsetzung des pCarlsberg 165 auf einer zweiten Rolle, aber vom selben Schreiber. Zur Pheros-Episode s. bisher H. DE Meulenaere, 'La légende de Phéros d'après Hérodote (II, 111)', CdE 28 (1953), S. 248-260; A.B. LloYd, Herodotus, Book Il. Commentary 99-182, Leiden 1988, S. 38-43; Kurzhinweis auf die demotische Version bei $\mathrm{K}$. RYHOLT, JEA 84 (1998), S. 151.

(8) Eine zweite, spätere Version mit relativ ähnlichem Inhalt bei Diodor I, 59. Die knappen Angaben bei Plinius, HN 36, 74 könnten sich, da explizit von Blindheit die Rede ist, ebenfalls hierauf beziehen, möglich ist dies auch noch bei Johannes Malalas; FgrH 609, F5. Dagegen ist an den bei LLOYD, Herodotus Book II. Commentary 99-182, S. 38 weiter genannten Stellen Josephus, AJ VIII, 6, 2; BJ V, 9, 4 für mich nicht ersichtlich, in welcher Verbindung zur Pheros-Geschichte sie stehen sollen.

(9) J. Baines, K. Donker van Heel, H.-W. Fischer-Elfert, 'Abnormal Hieratic in Oxford: Two New Papyri', JEA 84 (1998), S. 234-236. Für einige weitergehende Auskünfte danke ich Herrn Fischer-Elfert, der den Text in extenso edieren wird. 
«das Oberhaupt» aber auch eine ehrende Bezeichnung für besonders herausragende Personen. Als solche erscheint sie vor allem in der Aufzählung von verstorbenen Personen (in der Auflistung von Gräbern), wo neben $p 3$ hsy «der Gelobte» auch $p 3$ hri «das Oberhaupt» als hervorhebendes Epitheton von Personen erscheint, die im Bewußtsein der Nachwelt besonders bedeutsam und verdienstvoll erscheinen $\left({ }^{10}\right)$. Von daher wäre es zumindest nicht undenkbar, daß es sich tatsächlich in diesem Papyrus um eine Person handelt, die besonderes Ansehen genoß und zumindest einen gewissen «Nachruhm» hatte.

Nunmehr ist es Zeit, zu den griechischsprachigen Quellen überzugehen. An erster Stelle stehen soll der pRylands 63. Diese Handschrift überliefert die Reste eines Gespräches des Philosophen Platon mit ägyptischen Priestern. Dabei erklärt ihm ein gewisser Peteesis die Zuordnung der Vokale zu den Planeten, der Organe zu den Planeten und der Körperteile zu den Tierkreiszeichen $\left({ }^{11}\right)$. Die Lehrinhalte stehen prinzipiell durchaus in ägyptischer Tradition, speziell die Melothesie, d.h. die Zuordnung von Körperteilen zu Göttern oder Sternen ( $\left.{ }^{12}\right)$. Andererseits setzt die Erwähnung der Vokale bereits voraus, daß nicht die ägyptische, sondern die griechische Schrift verwendet wird. $\mathrm{DaB}$ hier ein relativ apokryphes Werk vorliegt, daß so nicht vor der Ptolemäerzeit entstanden sein kann, dürfte sicher sein $\left({ }^{13}\right)$. Dennoch bleibt die Frage interessant, wen man als angemessenes Pendant zu Platon auf der ägyptischen Seite angesehen hat. Alle Traditionen, die sich mit dem Aufenthalt Platons (sowie des Astronomen Eudoxos) in Ägypten beschäftigen $\left({ }^{14}\right)$, stimmen darin überein, daß er sich in

(10) J. QUAEGEBeUR, 'Les »saints « égyptiens préchrétiens', OLP 8 (1977), S. 129-143, bes. S. 141-143.

(11) Textedition J. de M. Johnson, V. Martin, A.S. Hunt, Catalogue of the Greek Papyri in the John Rylands Library, Manchester, vol. 2. Documents of the Ptolemaic and Roman Periods (nos. 62-456), Manchester/London 1915, s. inhaltlich W. GuNDEL, Dekane und Dekansternbilder. Ein Beitrag zur Geschichte der Sternbilder der Kulturvölker, Glückstadt/Hamburg 1936; ND Darmstadt 1969, S. 264.

(12) S. hierzu ausführlich J.F. QuACK, 'Dekane und Gliedervergottung. Altägyptische Traditionen im Apokryphon Johannis', Jahrbuch für Antike und Christentum 38 (1995), S. 97-122; unabhängig davon zeigt ägyptische Ursprünge der Melothesie auch Chr. LEITZ, Altägyptische Sternuhren, OLA 62, Leuven 1995, S. 3-55. Weiteres in meiner noch unpublizierten Habilitafionsschrift.

(13) Neben dem Alphabet könnte auch, allerdings weniger sicher, der Gebrauch des Zodiakos in diese Richtung sprechen, der in Ägypten bisher nicht vorptolemäisch belegt ist, für Mesopotamien nach neuesten Ergebnissen aber bereits im frühen 5 . Jhd. v. Chr. vorliegt, s. F. RochBerg, Babylonian Horoscopes, Philadelphia 1998.

(14) Über die Historizität von Platons Reise nach Ägypten gehen die Meinungen weit auseinander. Neben extrem ablehnenden Haltungen wie z.B. Th. HoPFNER, Orient und 
Heliopolis aufgehalten hat $\left({ }^{15}\right)$. Diese Stadt galt als Zentrum des geheimen Wissens der Ägypter. Somit kann man vermuten, daß auch Platons Gesprächspartner Peteesis (ägyptisch Petese) als Priester aus Heliopolis verstanden wurde. Die Kombination aus Namen, Herkunft und arkanen Kenntnissen spricht dafür, daß es sich tatsächlich um die bereits aus den ägyptischen Quellen bekannte Figur handelt. Der Ruhm des Petese als magisch fähiger Priester aus Heliopolis war also damals so ausgedehnt, daß man diese Figur für geeignet angesehen hat, als Wissensvermittler an den großen griechischen Philosophen dargestellt zu werden $\left({ }^{16}\right)$. Für die bisher in der Forschung vertretene Position, Petese sei nur eine analoge Bildung zum bekannteren Astrologen Petosiris bzw. sogar mit ihm identisch $\left({ }^{17}\right)$, sehe ich keinen Grund.

Größer werden die Probleme mit der nächsten Quelle. Bei Dioskurides, De Materia medica, werden einige Autoritäten genannt, aus deren Werken bestimmte Synonyme für Pflanzen gewonnen sind. Hier finden sich neben den möglicherweise nach Persien weisenden Namen des Ostanes und des Zoroaster $\left({ }^{18}\right)$ sowie Pythagoras auch zahlreiche Erwähnungen ägyptischer Propheten $\left({ }^{19}\right)$. Darüber hinaus wird an einer Stelle, nämlich De Mat.

griechische Philosophie, Der Alte Orient, Beiheft 4, Leipzig 1925 und recht verhaltenen Positionen wie J. KerschensteInER, Platon und der Orient, Stuttgart 1945 steht z.B. die positive Evaluation von F. Daumas, 'Sur l'origine égyptienne de la tripartition de l'âme chez Platon', in: Mélanges Adolphe Gutbub, Montpellier 1984, S. 41-54. S. zuletzt B. MAthIEU, 'Le voyage de Platon en Égypte', ASAE 71 (1987), S. 153-167; an von ihm nicht genannten Arbeiten s. vor allem noch W. und H.-G. GunDEL, Astrologumena. Die astrologische Literatur in der Antike und ihre Geschichte, Sudhoffs Archiv Beiheft 6, Wiesbaden 1966, S. 77-86.

(15) Besonders explizit sind hier Strabo, Geogr. XVII, 1, 29; Clemens v. Alexandria, Stromata, I, XV, 69, 1

(16) Plutarch, De Genio Socrat. 7 (578f.) gibt Chonouphis als Gesprächspartner Platons in Memphis an, Clemens von Alexandria, Stromata I, XV, 69, 1 dagegen Sechnouphis als Namen von Platons Lehrer in Heliopolis, während Konouphis (sicher nur abweichende Schreibung von Chonouphis; ägyptisch dürfte $k 3-n f r$ 'schöner Stier' zugrunde liegen) als ägyptischer Lehrer des Eudoxos genannt wird.

(17) So W. Kroll, 'Peteesis', RE XIX, 1, Stuttgart 1937, Sp. 1125; W. und H.-G. GUNDEL, Astrologumena, S. 81.

(18) Vgl. J. BideZ, F. CuMONT, Les mages hellénisés. Zoroastres, Ostanès et Hystaspe d'après la tradition grecque, Paris 1938. Allerdings gibt es auch komplizierte Querverweise nach Ägypten im Zusammenhang dieser 'persischen' Weisen, die z.B. Ostanes zum Priester in Memphis machen. Die Details bedürften weiterer Klärung, s. vorläufig meine Bemerkungen in 'Von der altägyptischen Textilfärberei zur Alchemie', im: B. KRULL (Hrsg.), Die Rolle des Handwerks und seiner Produkte in vorschrifihistorischen und schrifthistorischen Gesellschaften im Vergleich (im Druck in der Reihe Instrumentum Monographies).

(19) Die betreffenden Stellen sind bei Th. HoPfNER, Fontes Historiae religionis aegyptiacae, Bonn 1922-25, S. 202-207 gesammelt. 
Medica V, $98\left({ }^{20}\right)$, ein gewisser Peteesios als Gewährsmann genannt. Er

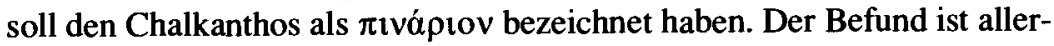
dings insofern kompliziert, als es sich um einen Satz handelt, der nur in den relativ jungen Handschriften $\mathrm{Di}$ und $\mathrm{H}$ steht, in den sonstigen Textzeugen jedoch fehlt $\left.{ }^{(21}\right)$. Man kann es dem Editor nicht verdenken, daß er diese schwach bezeugte Angabe in den Apparat verbannt hat. Jedoch dürfte sie auf jeden Fall auf eine antike Tradition zurückgehen. Weder ist plausibel, daß man im Mittelalter aus dem Nichts die korrekte Wiedergabe eines ägyptischen Namens erfunden, noch ein ägyptisches Wort kreiert hätte. Das Wort $\pi$ ivápıov wirkt aber schon aufgrund seines $p$-Anlautes gut ägyptisch. Der erste Teil dürfte als $p$ \} inr «der Stein» zu verstehen sein, auch wenn der hintere Bestandteil $\left({ }^{22}\right)$ noch Probleme bereitet $\left({ }^{23}\right)$. Die Angabe ist somit auf jeden Fall ernstzunehmen. Zu fragen wäre allenfalls, ob sie von Dioskurides selbst bzw. bald danach in den Text integriert wurde, oder ob es sich um eine späte Übertragung aus einer anderen, heute verlorenen Quelle handelt.

Die Details von Dioskurides Liste ägyptischer Ausdrücke bzw. von Decknamen der Propheten scheinen durchaus in einer echten ägyptischen Tradition zu stehen. Einerseits sind Decknamen in der ägyptischen Medizin prinzipiell mit einiger Wahrscheinlichkeit zu fassen $\left({ }^{24}\right)$, andererseits ist hier vor allem die bekannte Liste PGM XII 401-444 zu beachten, die mit den Angaben des Dioskurides engere Berührungspunkte aufweist $\left({ }^{25}\right)$. Bemerkenswert ist nun eine beiden gemeinsame Angaben, nämlich die Deutung des «Haares des Hundskopfaffen» als Dill (PGM XII, 419; Dioskor., De mat. med. I, 91). Gerade dieser Ausdruck ist nämlich als Bezeichnung einer offizinal verwendeten Pflanze tatsächlich in einem ägyptischen Text, dem pBrooklyn $47.218 .48+85,3,2$ bezeugt $\left({ }^{26}\right)$. Inwieweit dieser Petese als Gewährsmann des Dioskurides mit der Tradition

(20) In älteren Editionen wird dieser Abschnitt teilweise auch als Kapitel 114 gezählt.

(21) S. die Edition von M. Wellmann, Pedanii Dioscuridis Anazarbi De materia medica libri quinque, volumen III, Berlin 1914; ND 1958, S. 68.

(22) Dieser beginnt bereits mit dem $r$, da der Auslaut von inr 'Stein' in dieser Zeit im Ägyptischen bereits abgefallen ist.

(23) Im Prinzip würde man hier gerne zu etwas wie $\pi ı v \alpha \kappa \alpha \mu$ emendieren, s. die koptische Form a NIKaM (wörtlich 'schwarzer Stein') für Chalkanthos (Vitriol).

(24) S. hierfür zuletzt J.F. QUACK, OLZ 94 (1999), S. 460.

(25) S. die Bemerkungen von Th. HOPFNER, Griechisch-aegyptischer Offenbarungszauber, I, Leipzig 1921, § 491-499.

(26) J.F. QUACK, 'Das Pavianshaar und die Taten des Thot', SAK 23 (1996), S. 305-333, bes. S. $313 \mathrm{f}$. 
des hier untersuchten Magiers zu verbinden ist, bleibt unsicher. Immerhin stimmt nicht nur der Name überein, sondern die Beschäftigung mit den Kräften, welche die Natur bietet, würde ein verbindendes Glied schaffen.

Weitaus größer noch werden die Probleme hinsichtlich der nächsten und letzten großen Quelle, nämlich der alchemistischen Tradition. Zunächst sind hier die griechischen Texte in Betracht zu ziehen. In ihnen tritt die Gestalt eines gewissen Petasios $\left({ }^{27}\right)$ in folgenden Belegen auf:

- In der Auflistung der gebräuchlichen Fachausdrücke und Decknamen für die Substanzen wird zum «göttlichen Wasser», das dort als Quecksilber erklärt wird, als Quelle in der Version des ältesten Zeugen, Codex Marc. graecus 299 explizit Petasios angegeben (CAAG III, 15, 3) $\left({ }^{28}\right)$. Der Eintrag ist umso bemerkenswerter, als er der einzige Fall in der gesamten Synonymenliste ist, daß ein konkreter Autor als Beleg für die Auffassung angeführt wird.

- In der Auflistung der «Philosophen», die sich mit der Alchemie beschäftigt haben, wird unter anderem auch Petasios genannt (CAAG III, 26, 1) $\left({ }^{29}\right)$, wobei er hier in Nachbarschaft mehrerer anderer dem Namen nach eindeutig ägyptischer Autoren steht. Daneben erscheint in der Liste in der Version des Marcianus auch ein Isidoros $\left({ }^{30}\right)$, was quasi die Übersetzung von Petese «der, den Isis gegeben hat» ins Griechische darstellt. Eine Sonderlesart der Handschrift E macht Petasius zum König von Armenien $\left({ }^{31}\right)$, was kaum sonderlichen Quellenwert haben dürfte, und mutmaßlich einen Zusatz des Abschreibers

(27) Zu Petasios als Alchemisten s. M. BerThelot, Ch.É. Ruelle, Collection des anciens alchimistes grecs, Paris 1888; ND London 1963, 1, S. 68f. u. 201f.; E.O. v. LIPPMANN, Entstehung und Ausbreitung der Alchemie, Berlin 1919, S. 67f.; J. LINDSAY, The Origins of Alchemy in Greco-Roman Egypt, London 1970, S. 32f. u. 141f.; s. auch J. LETROUIT, 'Chronologie des alchimistes grecs', in: D. KAHN, S. MATTON (Eds.), Alchimie. Art, histoire et mythes. Textes et travaux de Chrysopaia 1, Paris/Mailand 1995, S. 11-93, bes. S. 47f. u. 88, der ihn etwa ins 4. Jhd. $n$. Chr. setzt. Diese Datierung ist für die vorliegende Untersuchung, in der es um Traditionen hinsichtlich einer Person, nicht um konkret zugeschobene Werke geht, ohne große Bedeutung, generell scheinen mir Letrouits Methoden z.B. hinsichtlich der ausschlieBlichen Fixierung auf den Codex $\mathbf{M}$ aber den Problemen nicht gerecht zu werden. DaB Petasius, da in einer Tradition über einen Weisen stehend, Deckname für ein pseudepigraphisches Werk sein könnte, wurde bisher in der Forschung nicht in Erwägung gezogen.

(28) Die anderen Textzeugen eliminieren dagegen den Namen, vermutlich durch eine Fehllesung bzw. im Versuch, aus dem unbekannten Namen ein vertrautes griechisches Wort zu machen, s. den textkritischen Apparat zur Stelle.

(29) Für die Lesungen des Codex M s. zusätzlich CAAG I, S. $110 \mathrm{f}$.

(30) CAAG I, S. 110f.; dieser Name fehlt in der Edition der Liste CAAG III, $25 f$.

(31) S. den textkritischen Apparat zu CAAG III, 26, 1. 
unter dem Eindruck des (ebenfalls sekundären) Titels des Kommentars des Olympiodoros, wie er in den Handschriften A und L bezeugt ist, darstellt.

- In einem Kommentar des Olympiodoros über Zosimus wird an zwei Stellen Petasios als Philosoph bezeichnet (CAAG III, 95, 15; 97, 17). Angegeben wird, daß er sich speziell mit dem Blei und seinen Eigenschaften beschäftigt hat. Eine dritte Erwähnung dieses Namens findet sich nur in der Lesart der Handschrift $L$ und dürfte sekundär sein (CAAG 98, 1 im textkritischen Apparat). Nach den Versionen der Handschriften A und $L$ (nicht jedoch der ältesten Handschrift $M$ ) ist das Werk insgesamt von Olympiodoros an Petasios, König von Armenien gerichtet $\left(^{32}\right)$. Da im Werk selbst Aussagen des Petasios zitiert werden, ist diese Angabe nicht originalgetreu $\left({ }^{33}\right)$, aber im Rahmen der vorliegenden Untersuchung als Zeugnis für das Nachwirken der Person sogar bei den Kopisten des späten Mittelalters nicht ohne Interesse.

- Relativ häufig erwähnt wird Petasios als Autorität im umfangreichen Kommentar des «Christen» (CAAG III, 278, 17; 282, 9; 416, 15) $\left({ }^{34}\right)$. Zum einen wird eine Schrift von ihm zitiert, in der er verschiedene mögliche Arten beschreibt, das alchemistische Werk zu vollbringen. Die Aussage »einige vollbringen die Iosis $\left({ }^{35}\right)$ in den Apparaten « scheint an einer Stelle von ihm selbst zu stammen (CAAG III, 278, 17f.). Jedoch wird durch die ausführlichere Parallelstelle CAAG III 282, 7-10 klar gemacht, daß diese Aussage an sich von Demokrit stammt und von Petasios dahingehend interpretiert wird, daß die betreffenden Personen durch die Apparate den Iochalkos hergestellt hätten. Die Schrift des Petasios zieht also (Pseudo)-Demokrit heran und ist eventuell sogar ein direkter Kommentar zu ihm, auf jeden Fall chronologisch jünger.

(32) CAAG III, 68 im textkritischen Apparat zu Z. 9. S. hierzu C. VIANO, 'Olympiodore l'alchimiste et les présocratiques: une doxographie de l'unité (De arte sacra, §18-27)', in D. KAhn, S. MATton (Eds.), Alchimie. Art, histoire et mythes. Textes et travaux de Chrysopoeia 1, Paris/Mailand 1995, S. 95-150; bes. S. 100f. Anm. 30.

(33) Letrourt, in: Alchimie, S. 47f. Anm. 155.

(34) Der Kommentar des «Christen» ist in Berthelots und Ruelles Edition sehr zerrissen, da sie die Fehlheftungen im Codex $M$ nicht ausreichend erkannt haben; s. dazu H. D. SAFFrEY, 'Histoire et description du manuscrit alchimique de Venise Marcianus graecus 299', in: D. KAHN, S. MATTON (Eds.), Alchimie. Art, histoire et mythes. Textes et travaux de Chrysopocia 1, Paris/Mailand 1995, S. 1-10.

(35) Die sogenannte «Rötung». Dies ist die von den Alchemisten angestrebte letzte Stufe der Färbung der von ihnen behandelten Substanzen. 
An einer weiteren Stelle wird Petasios zusammen mit Synesios lobend hervorgehoben. Er gilt als einer der später aufgetretenen Meister, die im Gegensatz zu den oft rätselhaften Alten die benutzen Verfahren klar und nachvollziehbar angegeben hätten (CAAG III 416, 12-22). Zu Petasios speziell wird angegeben, daß er klar und ausschließlich Arsen angegeben hätte (also keine Decknamen gebraucht) und Maßangaben und Kombinationen exakt genannt habe. Ferner erfährt man, daß er sich denen anschließt, welche die Formel »Die Natur freut sich an der Natur; die Natur triumphiert über die Natur « schreiben. Letzteres ist ein klarer Hinweis auf Pseudo-Demokrit, durch dessen Physika et Mystika sich dieser Ausspruch wie ein roter Faden zieht, wobei er angeblich als entscheidend wichtiger Lehrsatz des Ostanes im Innern einer Säule im Tempel (vermutlich von Memphis) gefunden wurde $\left({ }^{36}\right)$.

Eine weitere Erwähnung findet Petasios in einer umfangreicheren Zusammenstellung technischer Anweisungen und theoretischer Erörterungen über die Herstellung künstlicher Steine, die angeblich aus einem Buch stammt, das aus dem Sanktuar der Tempel stamme $\left({ }^{37}\right)$. Zitiert wird konkret aus einem Werk des Petasios, das »Demokritäische Denk-

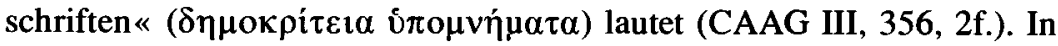
ihm soll Petasios die Terminologie der alten Autoren erklärt haben. Sowohl der Bezug auf Demokrit, der als gegenüber Petasios älterer Autor kommentiert wird, als auch das Bemühen um die Erläuterung schwieriger Ausdrücke passen zu dem, was bereits über Petasios gesagt wurde.

Ferner muß man noch den Brief des Ostanes an Petasios erwähnen (CAAG III 261, 9-262, 21) ${ }^{(38}$ ). Er beschreibt in komplizierter und bildhafter Weise, wie ein quasi allmächtiges »göttliches Wasser« erzeugt werden

(36) Demokrit und die Physika und Mystika sind oft besprochen worden, s. die 'klassischen' Studien von M. WellmanN, Die Georgika des Demokritos, APAW 1921, 4, Berlin 1921, ders., Die Фvбıká des Bolos Demokritos und der Magier Anaxilaos aus Larissa, Teil I, APAW 1928, 7, Berlin 1929 sowie A.-J. Festuglère, La révélation d'Hermès Trismégiste, I. L'astrologie et les sciences occultes, Paris ${ }^{2}$ 1950, S. 219-238, in neuerer Zeit etwa LinDSAY, Origins of Alchemy, S. 90-130; R. HALleuX, Les alchimistes grecs, tome 1. Papyrus de Leyde, Papyrus de Stockholm, recettes, Paris 1981, S. 62-75; I. VERENO, Studien zum ältesten alchemistischen Schriftum auf der Grundlage zweier erstmals edierter arabischer Hermetica, Islamkundliche Untersuchungen 155, Berlin 1992, S. 54-133; LETROUIT, in: Alchimie, S. 74-80. J. P. HerschBell, 'Democritus and the Beginnings of Greek Alchemy', Ambix 34 (1987), S. 5-20 diskutiert reale Bezüge von Demokrits Werk zu den alchemistischen Texten, S. 12-14 wird dort auch die konkrete Formel besprochen.

(37) CAAG III, 350,4-364,4.

(38) Generelle Bemerkungen und Neuedition bei BIDEz/CuMONT, Mages hellénisés, I, S. 208-210; II, S. 334-336. 
soll ( $\left.{ }^{39}\right)$. Dabei handelt es sich um das einzige erhaltene Schriftstück der griechischen Alchemie, dem tatsächlich Ostanes als Autor gegeben wird. Die Schlußworte des Textes zeigen eindeutig christliches Gedankengut, was wohl auch dazu geführt hat, daß dieser Brief als späte pseudepigraphische Schrift angesehen wurde $\left({ }^{40}\right)$. Jedoch besteht der Verdacht, daß es sich nur um den Zusatz eines späten Abschreibers handelt, jedenfalls fehlt der verdächtige Abschnitt vollständig in der Version des Codex Paris $2419\left({ }^{41}\right)$.

Unter Umständen ist Petasios auch in der arabischen Alchemie nachweisbar. In der Auflistung von Büchern im arabischen Fihrist findet sich unter den alchemistischen Werken auch ein »Buch des Dioskuros (ديسقرس), als Petasios ihn über die Probleme befragte « $\left({ }^{42}\right)$. Der Name erscheint dabei im Arabischen als بدسيوس (bdsyws). Die bereits von früheren Forschern vollzogene Gleichsetzung mit dem Petasios der griechischen alchemistischen Texte $\left({ }^{43}\right)$ ist lautlich plausibel $\left({ }^{44}\right)$. $\mathrm{Zu}$ befragen ist allerdings die Person des Dioskuros. Es besteht einige Wahrscheinlichkeit, daß es sich bei ihm um den aus den griechischen alchemistischen Quellen bekannten Priester des Sarapis handelt, der CAAG III 25, 9f.; 5669 (Dialog zwischen Synesios und Dioskuros); 199, 22; 211, 10; 432, 12 belegt ist. Dieser Dioskuros wird im allgemeinen entweder ins 4 . Jhd. $n$. Chr. datiert $\left({ }^{45}\right)$ oder für eine rein literarische Erfindung gehalten $\left({ }^{46}\right)$.

(39) Die Annahme von Bidez/Cumont, Mages hellénisés I, S. 210, im Text Spuren des alten dualistischen Weltbildes (sc. der Perser) wiederzufinden, möchte ich allerdings mit einem Fragezeichen versehen. Ebenso ist die II, S. 336, Bemerkung 5 geäußerte Behauptung, der geographische Horizont des Ostanes sei sehr begrenzt und schließe Ägypten nicht ein, insofern zu kurz gegriffen, als es dort nicht um geographische Kenntnisse im allgemeinen geht, sondern um Lagerstätten bestimmter Mineralien.

(40) So etwa von LeTrouIt, in: Alchimie, S. 48 u. 87 jeweils ohne Angabe von Gründen.

(41) S. den textkritischen Apparat bei Berthelot, Ruelle, CAAG III, S. 262 zu Z. 15.

(42) G. FuÜGEL, Kitab al-Fihrist, Leipzig 1871; ND Beirut 1964, S. 354, Z. 15.

(43) M. Berthelot, Les origines de l'alchimie, Paris 1885; ND Osnabrück 1966, S. 131; LeTrouIT, in: Alchimie, S. 48.

(44) F. SEzgIN, Geschichte des arabischen Schriftums, Band IV. Alchemie - Chemie, Botanik - Agrikultur bis ca. 430 H. Leiden 1971, S. 44f. will den Autor Mitāwas der arabischen alchemistischen Texten als Petasios erklären, was wegen der dann anzunehmenden erheblichen Korruptelen der Namensüberlieferung jedoch problematisch erscheint.

(45) So LeTrouIT, in: Alchimie, S. 47, die Existenz des Serapeums dürfte aber allenfalls einen Terminus ante quem angeben.

(46) So VERENO, Studien zum ältesten alchemistischen Schriftum, S. 19 Anm. 44 im AnschluB an I. HAMMER-JENSEN, Die älteste Alchymie, Kopenhagen 1921, S. 134f. Ich glaube aber nicht, daB in einem Lehrdialog innerhalb der 'arkanen' Naturwissenschaften der Fragende und Unwissende zu einer lächerlichen Figur gemacht wird; schon die zahlreichen hermetischen Dialoge sprechen gegen eine solche Wertung. 
Schließlich muß noch ein weiteres Problem der alchemistischen Texte angesprochen werden: Oben war bereits erwähnt worden, daß in der Version des Codex $\mathbf{M}$, im Gegensatz zu den sonstigen Textzeugen, in der Liste der Goldmacher auch ein gewisser Isidoros erscheint. Es wäre zu prüfen, ob dieser Isidoros eine ganz selbständige Figur ist, oder vielleicht nur eine andere Form des Petese darstellt, in welcher der Name nicht phonetisch wiedergegeben, sondern ins Griechische umgesetzt worden ist. Prinzipiell ist dies alles andere als sicher, da Isidoros ein häufiger Name ist $\left({ }^{47}\right)$. Außerhalb der Liste der Goldmacher erscheint Isidoros in den griechischen alchemistischen Texten nur einmal, nämlich bei Zosimos in der »Schlußabrechnung « $\left.{ }^{48}\right)$. Dort wird ein Buch erwähnt, das Hermes dem Isidoros gewidmet haben soll (CAAG III 242, 12). In ihm geht es um Färbungen, die früher als »natürliche Färbungen« bezeichnet wurden, in der Terminologie der jüngeren Alchemisten jedoch als "zeitgebundene

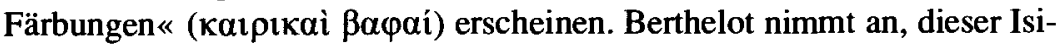
doros sei ein Synonym für Petesis $\left({ }^{49}\right)$. Sicher nachweisbar ist das nicht.

Noch weitaus skeptischer zu beurteilen sind die Versuche, Isidoros in der syrischen Alchemie wiederzufinden. Relevant sind folgende Textstellen: Im syrischen Lexikon des Bar Bahloul wird der Begriff $»$ Grab des/der $\left({ }^{50}\right)$ Isiodos « als »Mond oder Silber« erklärt $\left({ }^{51}\right)$. Berthelot denkt daran, Isiodos als Namen Isidoros »Gabe der Isis « zu erklären, alternativ erwägt er auch die Deutung als »Grab des Osiris « unter Verweis darauf, daß dieser Ausdruck bei Olympiodorus, CAAG II, 103 erwähnt wird. Gegen letzteres spricht allerdings, daß Osiris in der Alchemie im allgemeinen und auch an der vorliegenden Stelle als Symbol für Blei (bzw. auch Schwefel) dient. Gegen ersteres ist einzuwenden, daß eine derartige Verballhornung der Lautform zwar nicht ausgeschlossen ist, eine Bezeichnung als »Grab « einer menschlichen Person für eine Materialangabe aber sonst nicht üblich ist. Die bei weitem plausibelste Deutung ist meiner Ansicht nach »Grab

(47) RE IX, 2, Sp. 2060-2081 sind nicht weniger als 33 verschiedene Personen namens Isidoros behandelt, von denen aber keiner ein guter Kandidat für eine Identifizierung mit dem Isidoros der alchemistischen Texte ist.

(48) Zur 'Schlußabrechnung' s. vor allem die Übersetzung und Textetablierung bei FESTUGière, Révélation d'Hermès Trismégiste I, S. 275-282 u. 363-368, sowie M. MERTENS, Les alchimistes grecs, tome IV, ${ }^{\mathrm{Ie}}$ partie. Zosime de Panopolis, Mémoires authentiques, Paris 1995, S. LXV-LXVII.

(49) BeRTHElot, CAAG II, S. 233 Anm. 1.

(50) Die Genitivanknüpfung des Syrischen ist nicht im Genus flektierbar.

(51) M. BERTHELOT, La chimie au moyen âge, tome II. L'alchimie syriaque, Paris 1893; ND Osnabrïck/Amsterdam 1967, S. 122. 
der Isis «, da Isis in der griechisch-römischen Zeit tatsächlich eng mit dem Mond verbunden ist, der wiederum in der Alchemie mit dem Silber verbunden wird. Lautlich wäre die Verbindung um so plausibler, als in einer griechischen Fassung wohl $\tau \alpha \dot{\alpha} \varphi \circ \varsigma^{\prime \prime} I \sigma 1 \delta \circ \varsigma$ mit dem Genitiv 'I $\sigma \iota \delta o \zeta$ anzusetzen ist.

Aussichtsreicher scheint eine andere Stelle. In der syrischen Überlieferung des achten Buches des Zosimos wird angegeben, daß nach Isidos die Meditation das Werk vollbringe. Diesen Namen will Berthelot erneut als Isidoros verstehen und mit Petesis identifizieren $\left({ }^{52}\right)$. Zumindest der erste Schritt dieser Deutung ist insofern akzeptabel, als hier der Formulierung nach tatsächlich ein alchemistischer Autor vorliegen muß. Allerdings ist nicht auszuschließen, daß es sich doch erneut in Wirklichkeit um Isis handelt, da es ein ihr zugeschriebenes alchemistisches Werk, nämlich den Brief der Prophetin Isis an Horus gibt (CAAG III, 28-33 u. 33-35) $\left({ }^{53}\right.$ ). Eine Identifizierung des/der Isidos der syrischen Quellen mit Isidoros muß also zweifelhaft bleiben. Auch die Erklärung des letzteren als Peteesis kann zur Zeit nicht irgendwie abgesichert werden. Die folgenden Überlegungen werden sich also ausschließlich auf diejenigen Passagen der alchemistischen Texte stützen, in denen tatsächlich Petasios erwähnt wird.

Zunächst ist nochmals zu überlegen, ob die einhellige Annahme, der Petasios der alchemistischen Texte sei als ägyptischer Name Petese zu verstehen, korrekt ist. $\mathrm{Da} B$ Petasios im alchemistischen Korpus gelegentlich als König von Armenien bezeichnet wird, stellt jedenfalls kein Problem dar, da diese Angabe sich nur in spürbar sekundärer Form in jüngeren Handschriften findet. Die Hauptstütze für die positive Identifikation dürfte die Bezeugung bei Dioskurides darstellen. Sie zeigt Peteesios als jemanden, der Synonymbezeichnungen für Mineralien zusammengestellt oder zumindest eine ungewohnte (nämlich ägyptische) Terminologie für sie verwendet hat. Dieser Befund entspricht nun gut der Synonymenliste des alchemistischen Korpus, die Petasios als Autorität für einen mineralischen Stoff (nämlich das Quecksilber) nennt. Angesichts dieser frappanten Übereinstimmung kann man davon ausgehen, daß ungeachtet der leicht unterschiedlichen Vokalisationsform dieselbe Person gemeint ist.

(52) Berthelot, Chimie au moyen âge, II, S. 239.

(53) $\mathrm{Zu}$ diesem in zwei Fassungen überlieferten Werk s. besonders Festuglère, Révélation d'Hermès Trismégiste I, S. 253-260; LindSAY, Origins of Alchemy, S. 194-211; M. MERTENS, 'Une scène d'initiation alchimique: La 'lettre d'Isis à Horus', RHR 205 (1988), S. 3-23; VERENO, Studien zum ältesten alchemistischen Schriftum, S. 284-287. 
Ihr Name ist sicher ursprünglicher mit der Lesart der Dioskuridesüberlieferung als Peteesios anzusetzen $\left({ }^{54}\right)$, was eine der normalen griechischen Wiedergaben des ägyptischen Namens p3-čci-3s.t ist $\left({ }^{55}\right)$. Die Form der alchemistischen Papyri mag, sofern sie nicht rein sekundär ist, von konkret belegten Formen wie $\pi \varepsilon \tau \alpha$ jol $\zeta$ oder $\pi \varepsilon \tau \varepsilon \alpha \sigma i \zeta\left({ }^{56}\right)$ ausgehen.

Für diese Person ist ein Werk anzusetzen, daß sich mit Mineralien beschäftigte und dabei ungewöhnliche Ausdrücke als Synonyme für bekannte Sachen erklärte, dabei wenigstens teilweise ägyptische Namen zugrundelegte. Es ist plausibel, daß ein solcher Autor sich auch mit alchemistischen (bzw. chemischen) Fragen beschäftigt hat. Von daher kann man annehmen, da $B$ die sonstigen Bezeugungen der griechischen alchemistischen Texte sich ebenfalls auf diese eine Person beziehen. Auch die vom «Christen» hervorgehobene Tatsache, daß Petasios seine alchemistischen Werke klar und verständlich formuliert haben soll, paßt zu einem Schriftsteller, der sich um die Erklärung vorhandener Decknamen bemüht. Offenbleiben muß dabei allerdings zunächst, ob alle ihm zugeschriebenen Werke tatsächlich von ihm stammen, oder ob ihm pseudepigraphisch spätere Produkte zugeschoben wurden. Sofern die Notiz bei Dioskurides tatsächlich original ist, und nicht im Zuge der späteren Überlieferung aus einer anderen Quelle eingedrungen, hätte man für die (vergleichsweise echtesten) Schriften des Peteesios einen terminus ante quem von etwa 75 n. Chr. Etwaige chronologische Schlüsse aus der Position des Petasios als Kommentator Demokrits sind mit zu vielen Unsicherheiten behaftet, um sonderliche Beweiskraft zu haben. Unter der keineswegs sicheren Voraussetzung, daß der von Petasios kommentierte Text mit den heute überlieferten Physika et Mystica weitgehend identisch ist, und daß diese Physika et Mystica aufgrund der Erwähnung eines Metalls Klaudianon in die Kaiserzeit (Claudius oder später) datiert werden können, diese Erwähnung zudem zum Originaltextbestand gehört und nicht im Zuge einer späteren Überarbeitung eingedrungen ist $\left({ }^{57}\right)$, könnte man den zeitlichen Ansatz des

(54) Gerade in umgekehrter Richtung hat SALMASIUS, Plinianae exercitationes, 816 nach Angabe des kritischen Apparats bei WellmanN, Dioscuridis De materia medica, S. 68 die Emendation zu Petasios vorgeschlagen, mutmaBlich, um die Form an den Befund der alchemistischen Handschriften anzugleichen.

(55) F. PREISIGKE, Namenbuch, enthaltend alle griechischen, lateinischen, ägyptischen, hebräischen, arabischen und sonstigen semitischen und nichtsemitischen Menschennamen, soweit sie in griechischen Urkunden (Papyri, Ostraka, Inschriften, Mumienschildern usw.) Ägyptens sich vorfinden, Heidelberg 1922; ND Amsterdam 1967, Sp 312.

(56) F. PREISIGKE, Namenbuch, Sp. 309 u. 312.

(57) $\mathrm{Zu}$ den Problemen bei der Datierung des demokritäischen Werks s. die oben Anm. 35 genannte Literatur. 
Werkes relativ eng auf die Mitte des 1 . Jhds. $n$. Chr. festlegen. Sofern es sich nur allgemein um ein Demokrit zugeschriebenes Werk handelt, sind die Möglichkeiten erheblich vielfältiger.

Somit lassen sich zwei verschiedene Konzentrationspunkte ausmachen. Auf der einen Seite haben wir einen ägyptisch bezeugten Petese, Priester von Heliopolis und versierter Magier, der mutmaßlich auch als fiktiver Lehrmeister des Platon aufgefaßt wurde. Auf der anderen Seite steht ein Peteesios, interessiert an Mineralien und mutmaßlich auch an Alchemie, bzw. zumindest von Späteren als geeignete Autorität für die Zuschreibung von Werken angesehen. Es bleibt noch die Frage, ob eine Identifizierung dieser beiden Gestalten möglich oder sogar wahrscheinlich ist. Angesichts der relativen Häufigkeit des Namens kann hier keine absolute Gewißheit gewonnen werden. Andererseits ist es nicht a priori unwahrscheinlich, zwei Gestalten gleichen Namens, die beide Interesse in arkanen Bereichen zeigen, miteinander zu identifizieren. Die Werke des an Mineralien und Alchemie interessierten Petese sind allerdings Griechisch abgefaßt, während der ägyptische Priester Petese zu einer Zeit lebte, in der Verwendung des Griechischen nicht plausibel ist. Man müßte also entweder annehmen, daß seine Werke aus dem Ägyptischen ins Griechische übersetzt wurden, oder aber daß ihm pseudepigraphisch später Werke zugeschoben wurden. Letzteres muß sogar als fast sicher gelten, wenn man annimmt, daß der Demokrit-Kommentator Petasios mit dem Petese des abnormal-hieratischen Textes zu identifizieren ist, der vor der Lebenszeit sogar des echten Demokrit anzusetzen ist. Die Wahrscheinlichkeit eigener ägyptisch abgefaßter Werke ist auch insofern nicht allzu hoch, als in Ägypten technische Werke fast nie mit dem Namen eines konkreten menschlichen Autors versehen werden $\left({ }^{58}\right)$.

Immerhin gibt es einen sogar sehr berühmten Fall, bei dem mit Übergang des Sprachmediums von Griechisch zu Ägyptisch auch eine konkrete Autorenzuschreibung einher geht. Die bisher bekannten, weitgehend noch unveröffentlichten demotischen astrologischen Werke werden, soweit die Einleitung überhaupt erhalten ist, auf Imhotep zurückgeführt ${ }^{(59}$ ). Dieser ist zwar selbst ursprünglich ein Mensch, der erst sekun-

(58) Explizite Autorenangaben sind innerhalb der ägyptischen Literatur nur in den Lebenslehren üblich.

(59) Hier kenne ich derzeit vier demotische Handschriften, nämlich pCarlsberg 66, s. M. Chauveau, 'Un traité d'astrologie en écriture démotique', CRIPEL 14 (1992), S. 101105; bes. S. 102; pCtYBR 4222; unveröffentlicht, zitiert bei RYHOLT, Story of Petese, S. 81f. (dort ist der Name verlesen, meine Angabe beruht auf Kollation des Originals) sowie zwei Handschriften in Florenz. 
där vergöttlicht wird, für die Epoche der astrologischen Handschriften ist er jedoch sicher als offenbahrungbringender Gott zu verstehen $\left({ }^{60}\right)$. Dagegen erscheinen beim Übergang ins Griechische konkrete Autorenangaben. Besonders wichtig ist hierbei die Information des astrologischen Papyrus Salt (pLouvre N 2342 bis), der angibt, Petosiris und König Necheus $\left({ }^{61}\right.$ ) hätten hier Wissen von Hermes und Asklepios, welcher Imouthou, Sohn des Hephaistos ist, erhalten; d.h. also von den Göttern Thot und Imhotep dem Großen $\left({ }^{62}\right)$, Sohn des Ptah. Hier dürften mit einiger Wahrscheinlichkeit die bekannten astrologischen Autoren König Nechepso und Petosiris zu erkennen sein $\left({ }^{63}\right)$, die zur Entstehungszeit der astrologischen Werk als normale, wenn auch weise Menschen gelten, nicht als Götter. Ganz ähnlich gibt Firmicus Maternus, Mathesis IV, prooem., 5 an, Mercur hätte die Astrologie Aesculapius und Anubis(?) beigebracht und Petosiris und Nechepso hätten diese weiter erklärt. Entsprechend wäre es auch denkbar, daß technische bzw. alchemistische Werke, sobald sie nicht mehr ägyptisch (demotisch), sondern griechisch tradiert wurden, mit einem konkreten Autorennamen, u.a. eben dem des Peteesis versehen wurden.

Tatsächlich läßt sich sogar ein Beleg anführen, in dem ein solcher technischer Traktat schon in ägyptischer Sprache einem konkreten Autor zugeschrieben war, nämlich den pWien D 6614. In den Resten seines Titels $\left({ }^{64}\right)$ heißt es $\left({ }^{65}\right):[\ldots]$. iw $=f \operatorname{sh} 3 n w^{e}\left(\right.$ ?) $w^{i} b($ ?) $n$ ḥw.t-nčr $n$ 'Imn-r'-

(60) Vgl. D. WILDUNG, Imhotep und Amenhotep. Gottwerdung im alten Ägypten, MÄS 36, München/Berlin 1977.

(61) Diese Namensform, die besonders eng dem Namen des historischen Königs Necho der 26. Dynastie entspricht, ist auf jeden Fall ursprünglich und korrekt, bei E. RIESs, Nechepsonis et Petosiridis fragmenta magica, Philologus Supplement 6 (1891-1893), S. 331, Fr. 6, Z. 3 wird zu Unrecht eine Emendation zu Nechepso vorgeschlagen. Vgl. J. RAY, 'Pharao Nechepso', JEA 60 (1974), S. 255f.; R. Krauss, 'Necho II. alias Nechepso', GM 42 (1981), S. 49-60

(62) Das auslautende ov des griechischen Textes dürfte auf ägyptisches wr zurückgehen; in den ägyptischen Texten wird er ja sehr oft 'Iyl-m-htp wr s3 pth u.ä. genannt.

(63) W. und H. G. GundEL, Astrologumena, S. 26 u. 29.

(64) $\mathrm{DaB}$ es sich tatsächlich um den Titel des Werkes, nicht um das Kolophon eines Abschreibers handelt, ergibt sich eindeutig daraus, daB die betreffenden Zeilen als erste oben auf der Seite stehen, und der gesamte astrologische Text auf sie folgt.

(65) Der Text ist von E.A.E. REYMOND, From the Contents of the Libraries of the Suchos Temples in the Fayum, Part II: From Ancient Egyptian Hermetic Writings, MPER NS 11, Wien 1977, S. 143-157, T. VI sehr schlecht veröffentlicht und von G.R. HuGHES, 'An Astrologer's Handbook in Demotic Egyptian', in: L.H. Lesko, Egyptological Studies in Honor of Richard A. Parker, Hanover/London 1986, S. 53-69 (dort S. 69), sowie M. SMITH, 'Lexicographical Notes on Demotic Texts II', Enchoria 13 (1985), S. 111-114, als astrologisch erkannt worden. Ich versuche hier, anhand der veröffentlichten Photographie einen leidlich korrekten Text zu etablieren. 
nsw-n-n3-nčr.w n p3 nčr '3 [...] Imn-hns.w $r n$ n $m w . t=f$ mw.t-mht $w^{e}$ $s h 3$ pr- $n h$ " $[.$.$] . indem es geschrieben ist von einem(?) Priester(?) des$ Tempels des Amonrasonther, des großen Gottes [mit Namen..., Sohn des...]-Amun-Chons $\left({ }^{66}\right)$, der Name seiner Mutter ist Mut-em-het, ein Schreiber der Bibliothek. «

Ließe sich der aus dem alchemistischen Korpus bekannte Petasios also als Peteesis an eine bekannte Person außerhalb des alchemistischen Korpus anschließen, so gäbe es dazu noch eine Parallele gerade im ägyptischen Bereich. Im alchemistischen Schrifttum - sowohl in griechischer Sprache als auch in syrischer Übersetzung — wird gelegentlich ein Pibechios bzw. (fehlerhaft) Epibechios genannt. Diese Gestalt ist mutmaßlich mit dem Pibechios zu identifizieren, der PGM IV 3007 als Verfasser eines magischen Mittels gegen dämonische Besessenheit erscheint $\left({ }^{67}\right)$.

Möglicherweise können auch die Angaben über Pitys in den magischen Papyri, den vom Alchemisten Zosimos zitierten Autor Bitos sowie den bei Jamblich, De Myst. 8, 5; 10,7 genannten Übersetzer ägyptischer hermetischer Texte namens Bitys auf eine ägyptische Person namens pa- $t 3$ bezogen werden $\left({ }^{68}\right)$.

Diese Belege ergeben zusammengenommen immerhin einen Hinweis darauf, daß die am ehesten ägyptisch erscheinenden Personen innerhalb der griechischsprachigen alchemistischen Texte keine reinen Phantasiegebilde sind. Vielmehr stehen sie zumindest in einer größeren Tradition, die um den betreffenden Namen bzw. die Person den Ruhm von Kenntnissen in arkanen Bereichen gerankt hat. Es ist keineswegs auszuschließen, daß tatsächlich echte ägyptische Priester der Ausgangspunkt waren, die ihrer Mitwelt durch ungewöhnliche Taten besonders im Gedächtnis geblieben sind.

Schließlich wäre noch zu fragen, inwieweit Alternativen zur hier vorgeschlagenen Identifizierung der verschiedenen Petese-Gestalten bestehen. Zum einen ist ein Petese, Sohn des Erges (ägyptisch Herieu) als

(66) Ob es möglich ist, den Vatersnamen zu [č i $i]-1 m n-<w>-h n s w$ zu verbessern, was als Personenname in Theben in der Saitenzeit tatsächlich belegt ist, s. die Belege Demot. Nb. I, S. 1247?

(67) Zur Identität der beiden Personen s. etwa Lindsay, Origins of Alchemy, S. $153 \mathrm{f}$. und zuletzt LeTrouIT, in: Alchimie, S. 21 f.; zum Autor der PGM s. H.-J. THISSEN, 'Ägyptologische Beiträge zu den griechischen magischen Papyri’, in: E. Graefe (Hrsg.), Religion und Philosophie im Alten Ägypten, Festgabe für Philippe Derchain zu seinem 65. Geburtstag, OLA 39, Leuven 1991, S. 295.

(68) S. hierzu, mit Ausnahme des alchemistischen Belegmaterials, THISSEN, in: Fs Derchain, S. 295f.; Versuch einer Trennung dieser Gestalten bei LETROUIT, in: Alchimie, S. 4042; ablehnend zur Identifizierung auch MerTens, Zosime de Panopolis, S. 83. 
Hieroglyphengraveur aus dem griechisch und fragmentarisch auch in der demotischen Urfassung überlieferten Traum des Nektanebos bekannt $\left({ }^{69}\right)$. Er ist schon aufgrund seiner abweichenden Filiation als vom Magier Petese, Sohn des Petatum verschiedene Gestalt anzusehen. Als Identifikationsfigur für den Peteesios bzw. Petasios bei Dioskurides und in der Alchemie kommt er sachlich kaum in Frage.

Andererseits ist ein spezieller »Heiliger « Petese bekannt, dessen Verehrung bisher nur im Tempel von Dendur nachgewiesen ist $\left({ }^{70}\right)$. Diese offenbar stark lokal gebundene Gestalt kommt ebenfalls kaum für eine Identifizierung mit dem Petesis des Rylands-Papyrus, dem Petesios bei Dioskurides oder dem Petasios der alchemistischen Texte in Frage.

Da sich sonst keine weiteren Gestalten namens Petesis oder ähnlich als in der griechischen Kultur bekannt nachweisen lassen, besteht tatsächlich die Möglichkeit einer Traditionskette, die alle hier aufgeführten Stellen verbindet. Zusammengefaßt kann man folgende Entwicklung postulieren: Es gab in Ägypten Erzählungen um einen großen Weisen und Magier Petese, Priester im Tempel von Heliopolis. Diese Tradition könnte auf eine reale Person zurückgehen $\left({ }^{71}\right)$. Als Priester von Heliopolis wurde Petese wohl in der Ptolemäerzeit zum Lehrmeister Platons stilisiert. Schließlich rankten sich um ihn, möglicherweise ab ptolemäischer oder frührömischer Zeit, Zuschreibungen von Werken naturwissenschaftlichen bzw. alchemistischen Inhaltes. Wohl erst von mittelalterlichen Kopisten wurde diese Gestalt schließlich zu einem fiktiven König von Armenien gemacht.

Joachim Friedrich QuACK

(69) Griechischer Text ediert von U. WILCKEN, Urkunden der Ptolemäerzeit (Ältere Funde), Erster Band. Papyri aus Unterägypten, Berlin/Leipzig 1927, S. 369-374, Teilphotographie bei M. RAVEN, Papyrus van Bies tot Boekrol, Zutphen 1982, S. 58f.; an neueren Kommentaren s. etwa W. CLARYSSE, "«De droom von koning Nektanebo» op een griekse papyrus (U.P.Z. 81)', in: K.R. VEENHOF (Ed.), Schrijvend verleden, Zutphen 1983, S. 367 371; L. KoENEN, 'The Dream of Nektanebos', BSAP 22 (1985), S. 171-194; zur demotischen Version s. K. RYHOLT, 'A Demotic Version of Nectanebo's Dream (P. Carlsberg 562)', ZPE 122 (1998), S. 197-200. S. zuletzt J.-D. GAUGER, 'Der "Traum des Nektanebos" - die griechische Fassung', in A. BLAsIUs / B.V. SCHIPPER (Hrsg.), Aponalyptik und Ägypten. Eine kritische Analyse der relevanten Texte aus dem griechisch-römischen Ägypten, OLA 107 (Leuven/Paris/Sterling 2002), S. 189-219; K. RYHOLT, 'Nektanebo's Dream or the 'prophecy of Petesis', in BLASIUS/SchIPPER, Apokalyptik und Ägypten, S. 221-241.

(70) A.M. Blackman, The Temple of Dendûr, Kairo 1911, bes. S. 82-84.

(71) Gerade angesichts sonstiger Erfahrung mit ägyptischen Weisen der Tradition spricht einiges dafür, s. etwa Imhotep oder Amenohotep, Sohn des Hapu, die auch real existiert haben und auf die spätere Tradition als große Weise Eindruck gemacht haben. 